\title{
Design and Implementation of 3D Qipao Display System Based on Virtual Reality Technology
}

\author{
Yan Jiang ${ }^{1}$, Ruiliang Guo ${ }^{2}$ and Jing $\mathrm{Hu}^{3}$ \\ ${ }^{I}$ Computer Information Center, Beijing Institute of Fashion Technology, Beijing, 100029, China \\ ${ }^{2}$ Fashion Art and Engineering, Beijing Institute of Fashion Technology, Beijing, 100029, China \\ ${ }^{3}$ Department of Fine Arts, Artistic Vocational College of Anhui, Hefei, 230001, China
}

\begin{abstract}
Virtual reality technology has the realistic significance in the applied research of the garment field. In this paper, we present the central application fields for cloth simulation. Furthermore, describe the method for design and implementation the 3D Qipao Display System. This System can fully demonstrate the characteristics of patterns of the classic Qipao. In contrast with the traditional display approaches, the virtual garment display system based on virtual reality technology shows the better intuitional and interactive visual effect.
\end{abstract}

Keywords: Virtual reality, Qipao, Interactive technology.

\section{INTRODUCTION}

The problem of simulating the behavior of clothes is one subject of the computer graphics community, which can be used in virtual try-on system, digital films, games and Ecommerce applications. The challenges of cloth simulation are numerous, and have attracted research efforts for more than two decades. Compared with the rigid objects, flexible objects just as textile are inherently more difficult to model. Cloth is characterized by strong resistance to stretch and weak resistance to bending, which leads to a stiff set of equations and thus prohibits the use of large time step. Over the last decade a great deal of researches have been dedicated to implement cloth simulation. Some of them aim to reproduce the physical behavior of cloth [1]. Even more accurate ones $[2,3]$, and others are able to context of interactive VR (Virtual Reality) applications or games [4]. Recently, some outstanding productions have been published, and used successfully in Europe textile and garment enterprises [5-7].

With the continuous maturity and promotion of Virtual Reality Technology, a new teaching model comes forth in the current educational sector, which is highly reputed as "Virtual Reality Interactive Teaching". The 3D Display System based on Virtual Reality Technology and recommended in this paper has the vital realistic significance as it can facilitate the clothing majors to have the overall study and understanding of the cutting and modeling features of the classic models of Qipao through the 3D display in the system and also provide the platform to the people with strong interest in the Qipao Culture. So in contrast with the traditional display approaches, say, the literal description and photos, the virtual garment display system based on Virtual Reality Technology shows the better intuitional and interactive visual effect.

\section{THE APPLICATION OF 3D CLOTH}

There is a great potential for cloth simulation in several areas: from computer graphics to industrial $\mathrm{CAD}$ and prototyping applications, the entertainment industry and Ecommerce. In particular, cloth simulation can be a real benefit for the garment industry: visualizing a garment without actually making it drastically simplifies the prototyping phase, a task which usually involves significant expenditure on material resources and is time-consuming, requiring manual measuring, cutting, seaming etc. Some of the major applications are as follows.

\subsection{Garment CAD/CAM}

Modeling tools of clothing can allow fashion designers to experiment easily with a variety of fabrics and patterns on a 3D dynamic virtual mannequin before the actual garment is manufactured. Once the design is complete, it can be sent to a computer-controlled fabrication machine for weaving the cloth and cutting out the appropriate patterns. Potential buyers may even try the virtual garment before actually ordering it, using an augmented reality system. In this way customized clothing can be designed and made in an automatic fashion [8], shown in Fig. (1).

\subsection{Game Engine}

In computer animation and entertainment industry, the demand for more realistic virtual actors increases the need for more convenient tools which are faster,easier and better 


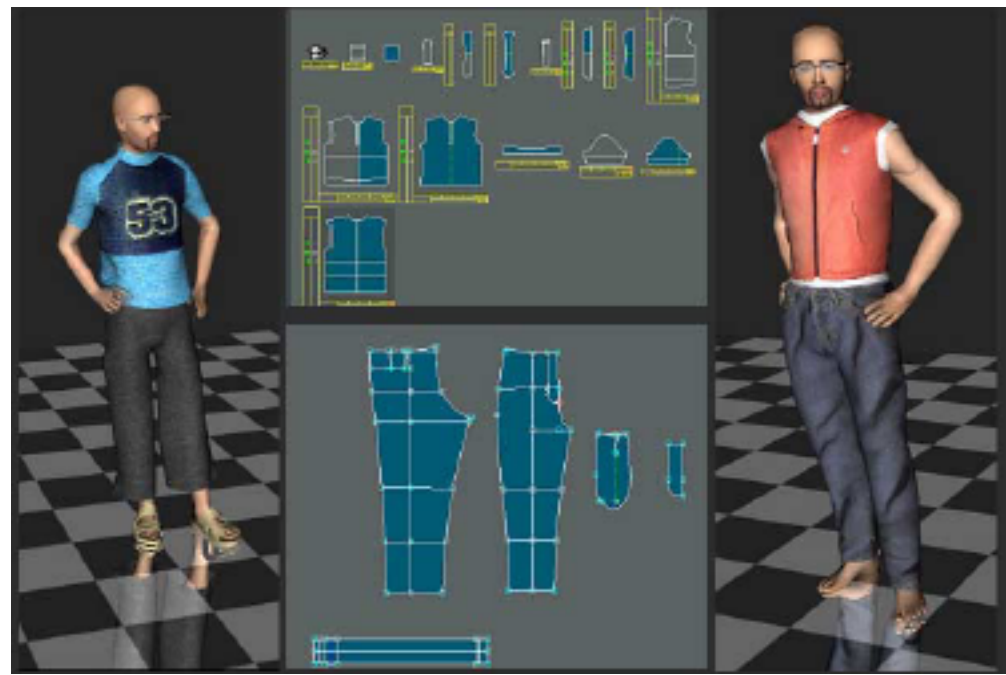

Fig. (1). Patterns for virtual garment.
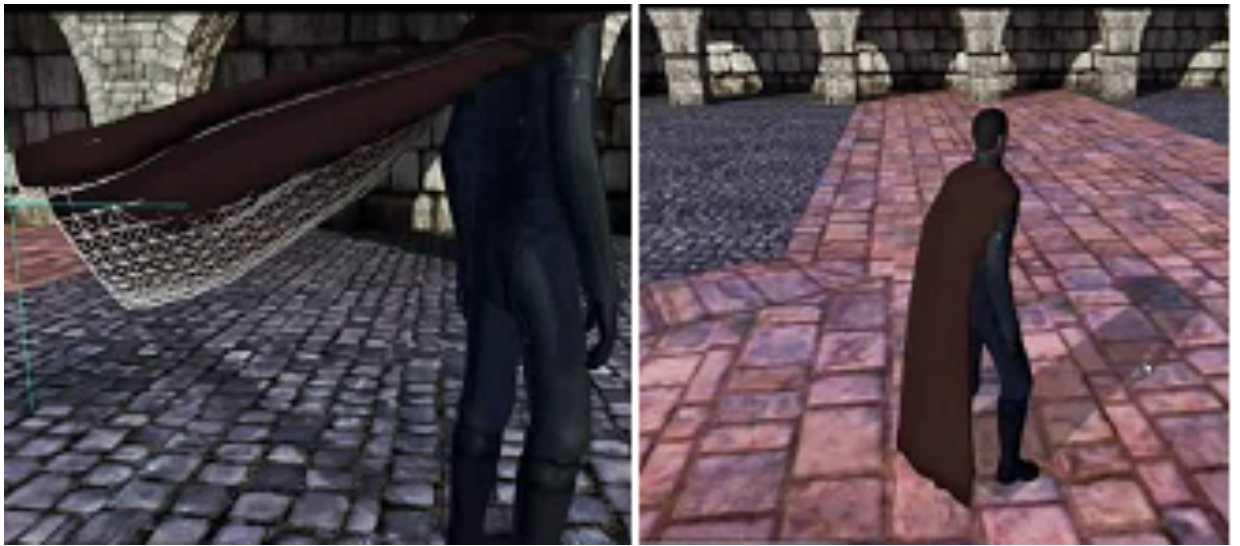

Fig. (2). The Snapshots of Havok Cloth demo.

at designing clothing on game characters. Just about it, many companies have enhanced the station of cloth simulation in general game engine. Many techniques have been developed and have moved from research labs to the animation studios. NVIDIA's cloth simulation provided unprecedented realism in many games, giving players a true next-generation gaming experience[9]. Another typical one is Havok. Use Havok Cloth to quickly and easily animate character clothing such as shirts, trousers, skirts, capes and ponytails, as well as environmental cloth. Havok products have been used in more than 170 of the world's best known game titles, including Halo 3, Halo 2, Stranglehold, BioShock, Crackdown, MotorStorm, and many more.

\subsection{E-Commerce}

With the Internet explosion and web based browsers, concepts like E-commerce are becoming popular. In this context, cloth and garment modeling offer a variety of opportunities for a typical business to customer, interactive E-
Commerce. A number of initiatives have arisen recently across the world [10-12], revolving around the concepts of Made-to-Measure manufacturing and shopping via the Internet. These initiatives are fueled by the current Web technologies available, providing an exciting and aesthetically pleasing interface to the general public, shown in Fig. (3).

The visitor to the online virtual store follows several successive steps provided [13]:

1. Select the garment items through the $2 \mathrm{D}$ garment catalog.

2. The requested garments and motion data are downloaded and are then loaded into the scene.

3. The user continues to the body measurements page containing a number of fields that are adjustable.

4. Finally, a trigger action starts the execution of realtime simulation. While the skeleton animation player updates the angle values of the mannequin's skeleton, the skin and clothes are deformed accordingly. 

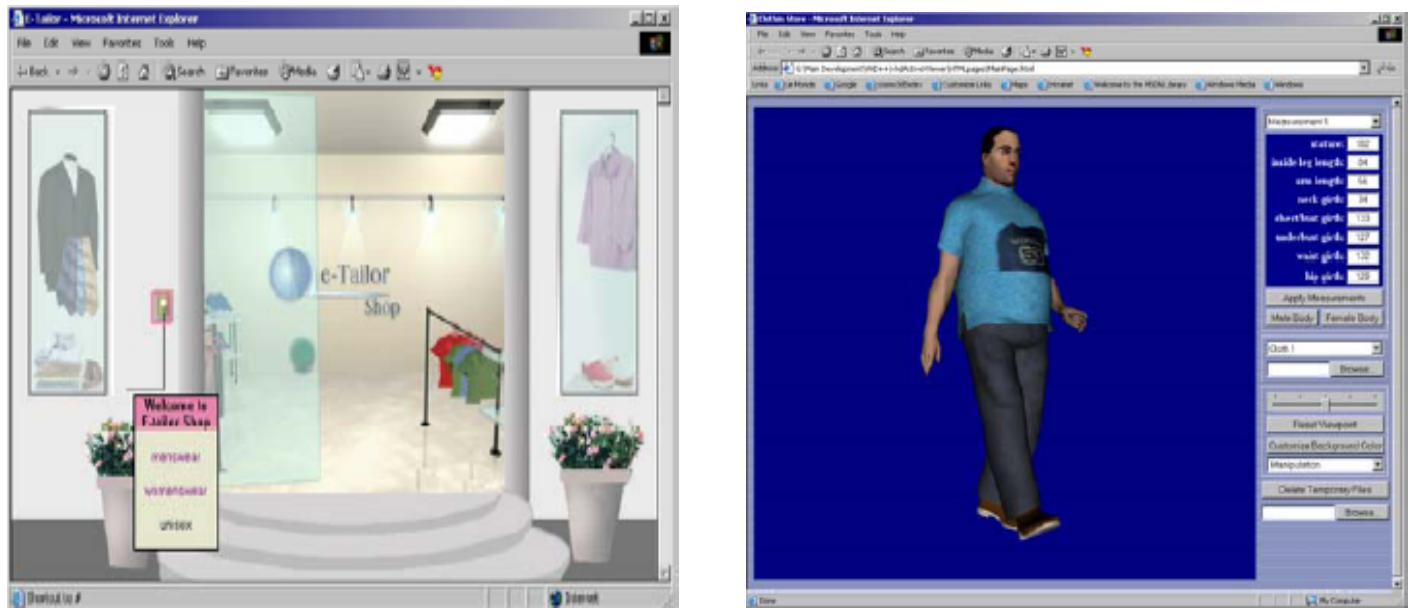

Fig. (3). The online clothing store.
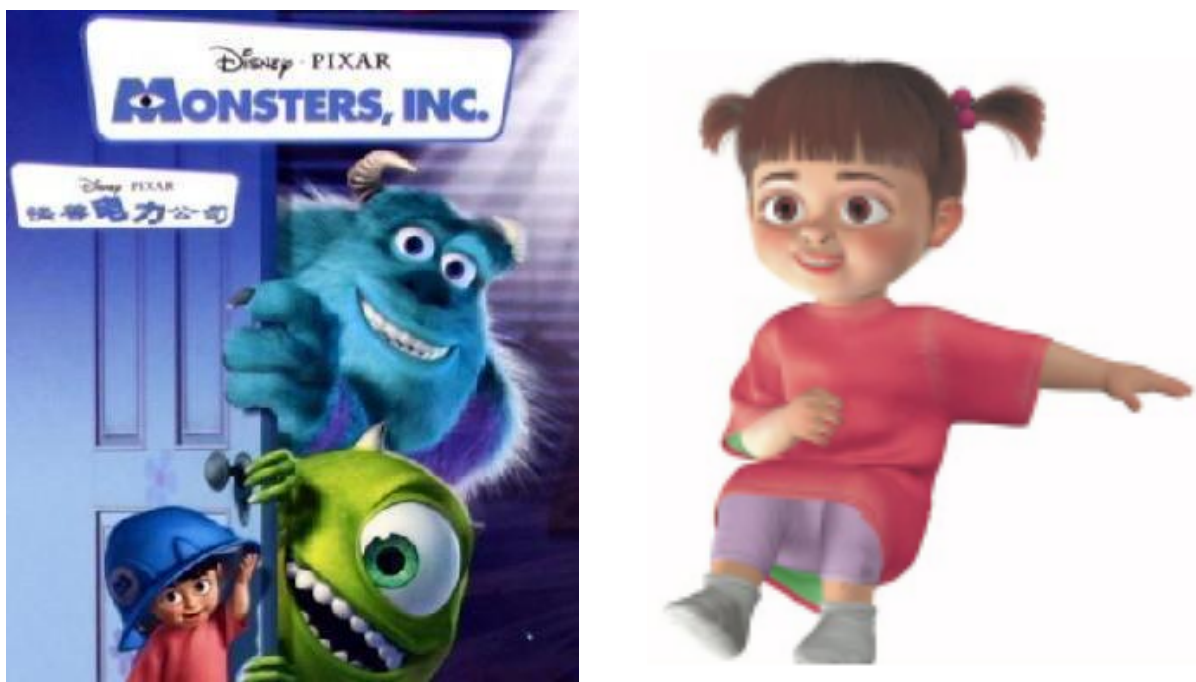

Fig. (4). Characters in Monsters, Inc.

\subsection{Computer Films and Fashion Shows}

Producing computer animation sequences is the most natural use of the computer simulation system. It is arguably also the easiest, as the accuracy needs only to satisfy visual realism, rather than quantitative mechanical precision. During latest decennary, the up to date cloth simulation algorithms have been used extensively for dynamics simulations of cloth and fur in Pixar Animation Studios [14-16]. The film "Monsters, Inc." produced by Pixar, shown in Fig. (4), was nominated 2001 Oscar best animated feature film.

In the context of Germanys CPD Fashion Fare 2004, the virtual prototyping process was tested for Modee's Spring/Summer Collection 2005 [17], shown in Fig. (5). As a consequence, prototyping is based on $2 \mathrm{D}$ patterns and technical sketches. For this approach the patterns have been created by the company itself with a commercial CADSystem and directly imported into the professional software.
In order to obtain appropriate physical parameters and simulate accurately the garments, fabric samples for each style has been featured in advance.

\section{SYSTEM DESIGN}

\subsection{Content Design in the System}

The major contents involved with the display system include Qipao-related theories, Qipao Styles, Qipao fabric and processing skills. Such knowledge points will be clearly illustrated in the system structure diagram in Fig. (6) in the system:

\subsection{Color Design in the System}

The significance of the color in the display system design should not be underestimated as it serves not only as a 


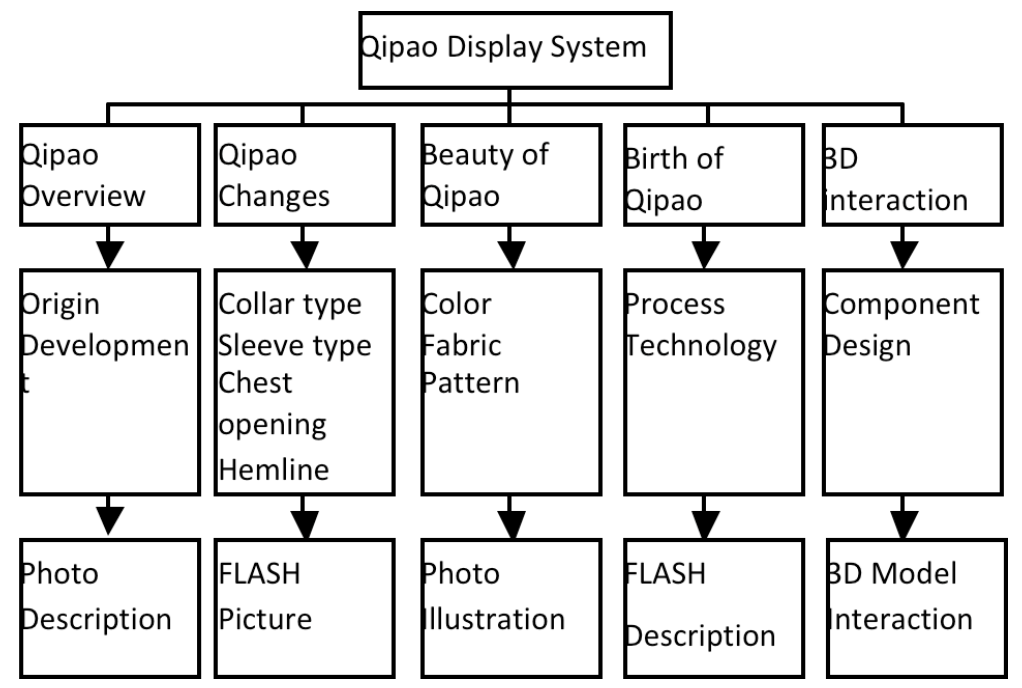

Fig. (6). System structure diagram.

specific visual symbol but also an approach for better organizing the system information and the formation of the excellent system format $[18,19]$. Not just for all these, the strongly designed color can additionally attract the users, arousing their interest. So in view of the choice of colors, it is important to identify the target and task for the color use, namely the communication assistance function of colors to facilitate the effective transmission of the information from the machine to the task.

This system is mainly intended to better promote the Chinese traditional Qipao for the purpose of the systematic study, inheritance, protection and development of Qipao, so this system adopts the popularly-accepted warm yellow hue as the basis supplemented with Purple and Tan to highlight the deep visual perception of its dignity and simplicity. The video information background inserted in the system is mainly involved with the traditional Chinese ink-and-wash paintings in clear and elegant hue.

\subsection{Interface Design in the System}

The interface design consists of four major Areas, respectively Title Area, Content Display Area, Interactive Control Area and Navigation Area, all of which are the Button Design for the purpose of simplicity, beauty and the clearly definitive target. Interactive function is designed in the form of Qipao teaching contents and display models. The interface design for Content Display Area adopts the left-right sliding type to pave the way for the pending mobile platform APK support in later development. Navigation Area is composed of five separate parts, respectively Qipao Overview, Qipao Changes, Beauty of Qipao, Birth of Qipao and 3D interaction, so each column has the second-level menu.

\subsection{Virtual Figure Design in the System}

This system is the one to display the traditional Chinese Qipao, so the leading virtual figure thus identified is the female image of the typical oriental features. The body proportion should be complied with the standards of the oriental females in the external appearance so as to bring into full play the features and styles of the traditional Qipao and bring the personality and the entire color of the system into the total unity with the system.

\section{KEY TECHNOLOGY OF THE SYSTEM IM- PLEMENTATION}

\subsection{CLO 3D Qipao Making}

The system uses the professional clothing Modeling Software CLO 3D for the Qipao style design. It is necessary to launch the Avatar after the design of the virtual model with the oriental features modeled upon true person for design and conduct the $3 \mathrm{D}$ model clothing wearing flow after dealing with the size change of the fitting model and the clothing trial dynamic simulation effects.

After the 2D template diagram is well drawn shown in Fig. (7), the 2D template is put on the model, so the template can be directly compatible with the 3D Simulation System and also with other CAD template format and then it can be imported into 3D Simulation System via DXF. In combination with the Avatar, it is proper to adjust the 2D plane model Sewing and continue to direct it until the lofting of the symmetric model. With a view of highlighting the fabric simulation result, the fabric texture may be used in the clothes by using the editing tools for correcting the pattern and direction. As Qipao belongs to the clothes of the independent structure, there is no need to re-design the hierarchical effect of dressing and it will be delicately polished up after being imported into 3DS MAX.

\subsection{Baking}

After the clothes are delicately processed in 3DS MAX, it is necessary to start the baking and the optimal operation of the virtual figure and the scene. Baking is to increase the 


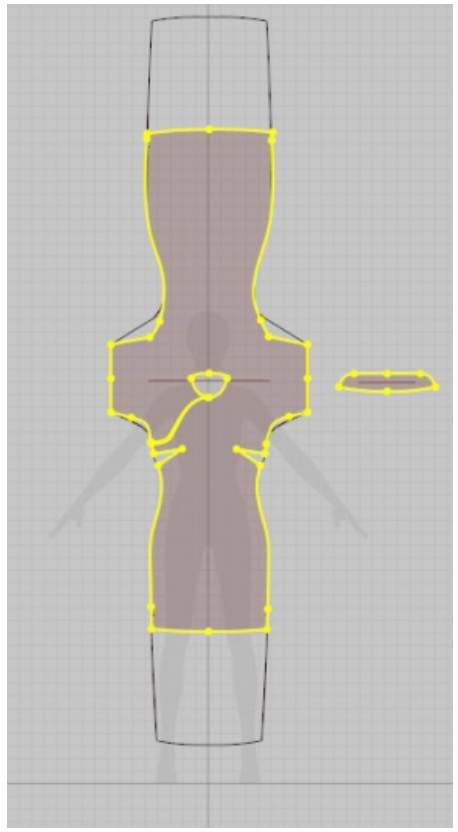

Fig. (7). 2D pattern adjustment.

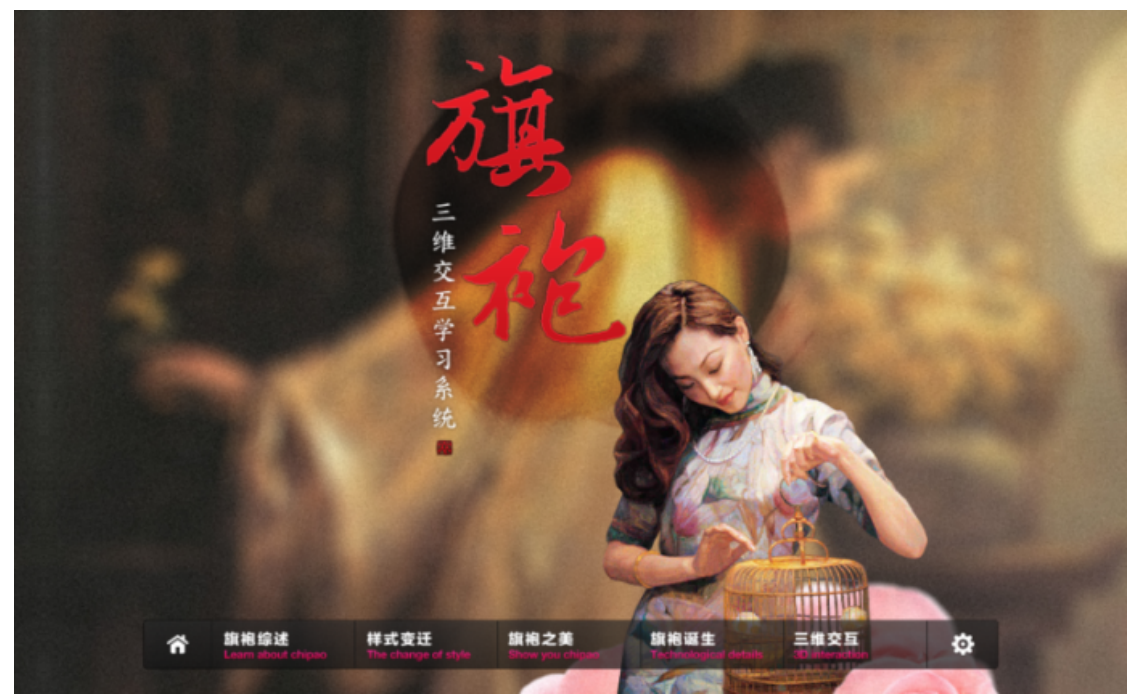

Fig. (8). Main interface of the display system.

sense of reality so as to transform the real-time light and shadow effect in 3DS MAX into the non-real-time effect in the VRP editor and to obtain the VR scene with the true effect. If the faces of the model in operation are too numerous or the document is too large in file size, it may lead to the slower system performance and it may take longer time for the users to download it after it is released online. The factors ultimately affecting the speed of the system operation is the faces and total numbers of the models in the scene and the total mapping volume in the virtual system scene model. If the number of models are too numerous, it may affect the speed to open the system scene and the export of the scene, therefore it necessitates the rational combination of the models.

\subsection{VRP Editing}

After the completion of the scene baking, "VRPlatform" in the "Tool" Command Panel is applied to import the scene into VRP-Builder Editor. VRP Editor may conduct the correction and editing of some effect of the model mapping in the scene, say, by adding the reflection effect, the setting of transparency effect and the adjustment of the degree of shadow, etc. It is necessary to start the "Script Editor" in VRP Editor and click "Insert Statement" Button, and "VRP Command-line Editor" would pop out, so now it is necessary to select the command name and set parameters to add the interactive script for the button, photo or module, thus implementing the interactive function of the VRP scene. 


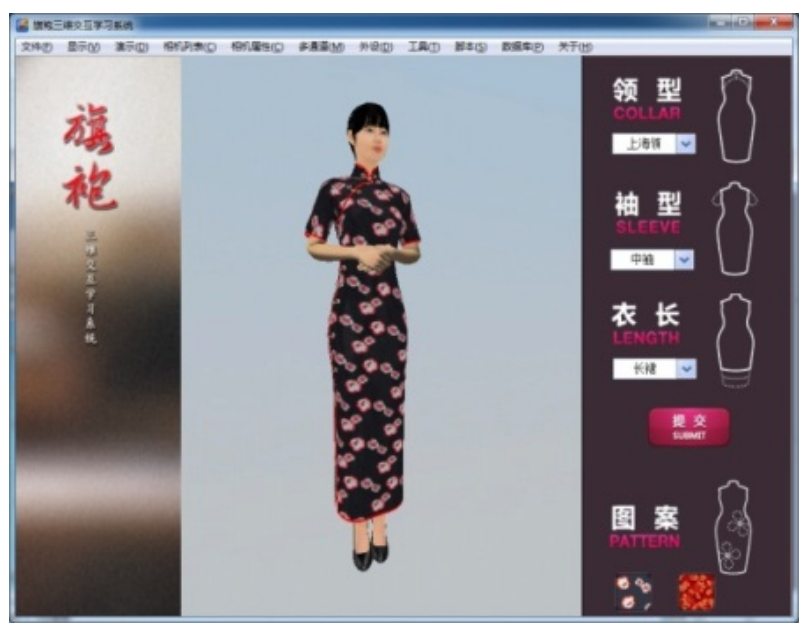

(a) Qipao in different styles

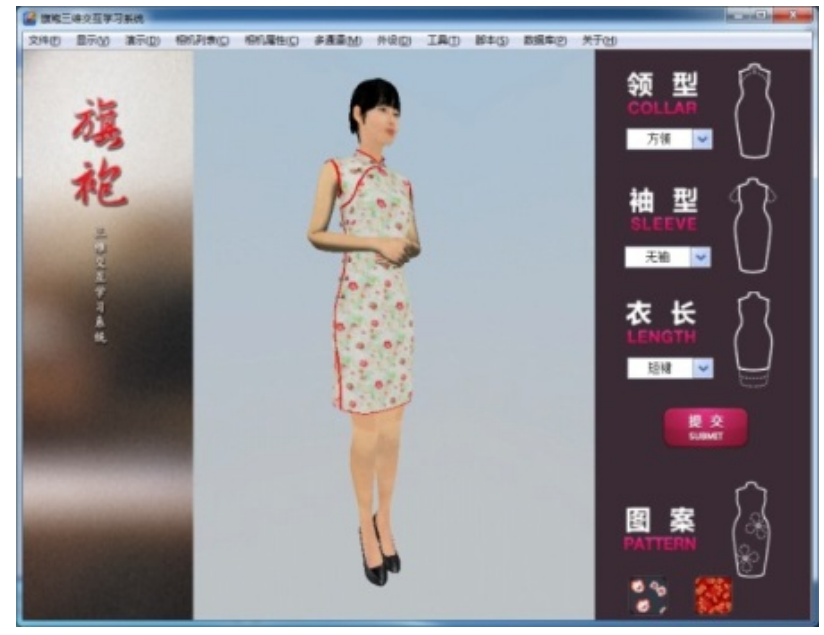

(b) Qipao in different patterns

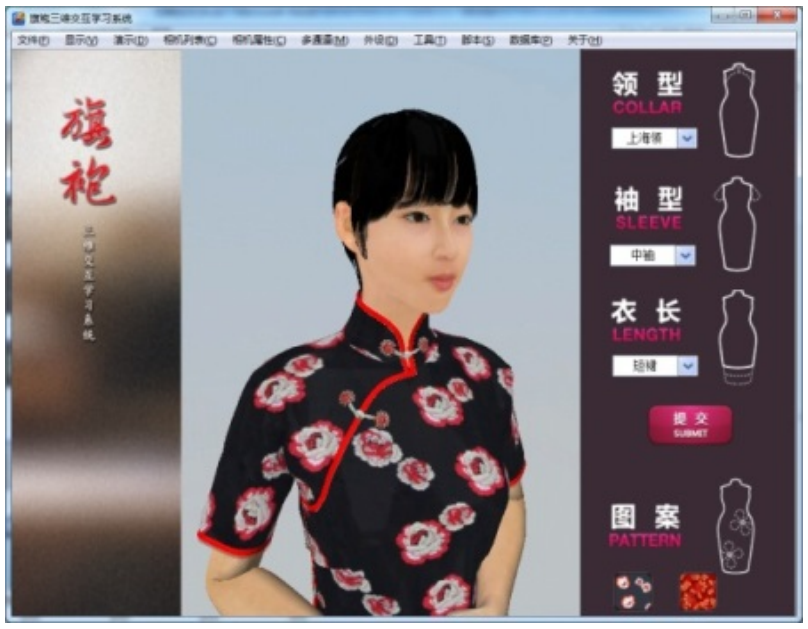

(c) Detailed Display of Qipao ornamental frogs

Fig. (9). Contd... 


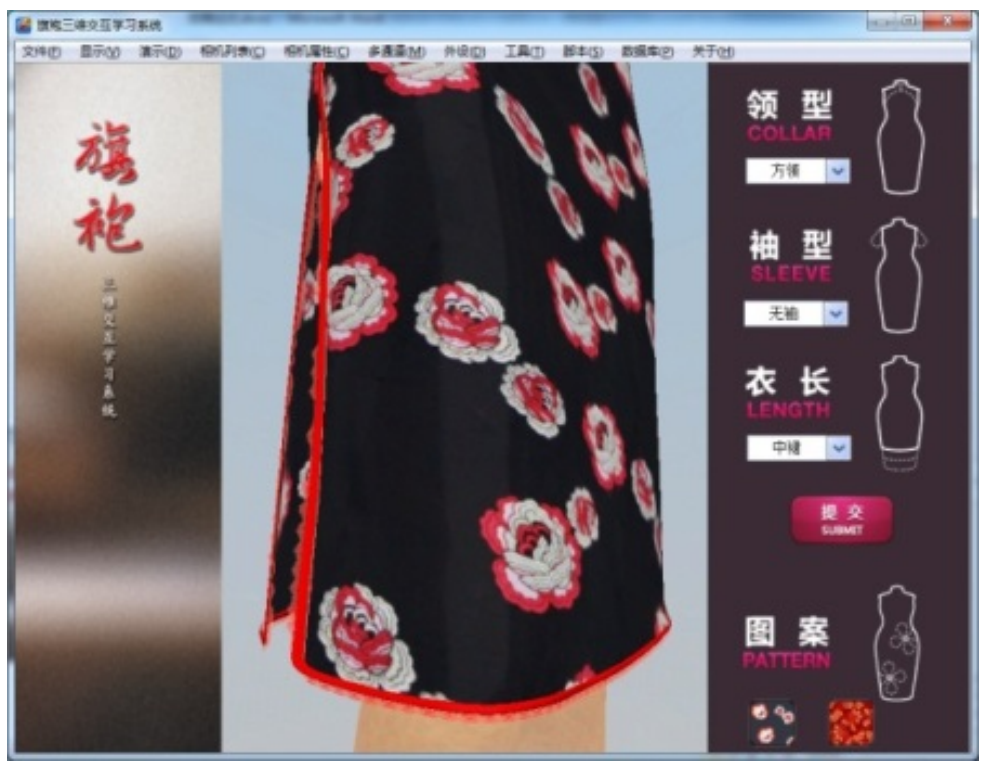

(d) Detailed Display of Qipao embroidered fringes

Fig. (9). Display of 3D interactive function.

\subsection{Testing and Evaluation}

The testing of the 3D Qipao Display System based on Virtual Reality Technology is mainly to conduct the test of the system display and the interactive function, and the system interface is illustrated in Fig. (8) and Fig. (9).

\section{CONCLUSION}

Cloth simulation techniques are related to a wide range of new applications such as virtual try-on, web applications, games, autonomous virtual worlds, etc. This encourages the researcher to create an ideal system offering both accurate simulation and real-time animation opens the field of new interactive virtual garment design and simulation applications, that both offer the accuracy needed by the garment industry and the interactive features required for virtual prototyping and visualization.

This paper designs and implements a 3D Qipao display system based on Virtual Reality Technology by comprehensively applying Qipao Design Science, Qipao Technology, Virtual Reality Technology, Clothing 3D visual technology, digital media technology. The software system has been verified by Copyright Protection Center of China (CPCC) and has obtained the computer software copyright registration (Registration No. 2013SR117887). This system has expanded the forms for Qipao design and display and also provided a platform for the general Qipao enthusiasts for specific learning. At the same time, the Virtual Reality Technology adopted in the system implementation process can be applied in other similar disciplines, thus enjoying the extensive development space.

\section{ABOUT AUTHOR}

Yan Jiang received the $\mathrm{Ph} . \mathrm{D}$. degree in textile material and textiles design at Tianjin Polytechnic University in 2010.
At present, she is an associate professor in the computer information center at Beijing Institute of Fashion Technology in China. Her main research interests lies on cloth simulation, virtual reality, and digital media technology.

Ruiliang Guo received the M.S. degrees in clothing engineering at Beijing Institute of Fashion Technology in 2003.

He is currently an associate professor in the department of fashion art and engineering at Beijing Institute of Fashion Technology, directing the clothing CAD laboratory.

Jing Hu completed her undergraduate at Hefei University in China (2006), and received the M.S. degrees in digital media technology at Beijing University of Technology in 2013. She is currently a lecturer in the department of fine arts at Artistic Vocational College of Anhui.

\section{CONFLICT OF INTEREST}

The authors confirm that this article content has no conflicts of interest.

\section{ACKNOWLEDGEMENTS}

This paper belongs to the project of the "High-level Talents Introduction and Training Program of Beijing Colleges and Universities (No. CIT\&TCD201404025)"; "Funding Project for Science and Technology Project of Beijing Municipal Education Commission".

\section{REFERENCES}

[1] P. Volino, N. Magnenat-Thalmann, "Comparing Efficiency of Integration Methods for Cloth Animation”, Proceedings of Computer Graphics International 2001, Hong-Kong, 2001, pp.265-274.

[2] Eberhardt, O. Etzmuß, M. Hauth, "Implicit-explicit Schemes for Fast Animation with Particle Systems", Eurographics Computer Animation and Simulation2000, Berlin Heidelberg, 2000, pp.137151. 
[3] Kwang-Jin Choi, Hyeong-Seok Ko, "Stable but Responsive Cloth", Computer Graphics, 2002,21(3),pp. 604-611.

[4] Young-Min Kang, Hwan-Gue Cho, "Bilayered Approximate Integration for Rapid and Plausible Animation of Virtual Cloth with Realistic Wrinkles", Computer Animation 2002, Geneva, Switzerland, 2002, pp.203-210.

[5] N. Magnenat-Thalmann, P. Volino, "From Early Draping to Haute Couture Models:20 Years of Research", Visual Computer,2005,21,pp.506-519.

[6] Louise Guay, http://www.myvirtualmodel.com.

[7] Uwe Rotter, http://www.fashion-me.com.

[8] P. Volino, N. Magnenat-Thalmann, "Accurate Garment Prototyping and Simulation", Computer Aided Design \& Applications, 2005, Vol. 2, pp. 1-4.

[9] Cyril Zeller, "Cloth Simulation(White Paper) ",NVIDIA Corporation, 2007,pp.1-9.

[10] Fashion on line, http://www.atc.gr/fol/index.asp

[11] Digital Fashion ltd., http://www2.dressingsim.com/
[12] Nordstrom, http://www.nordstrom.com

[13] A. Cordier, H. Seo, and N. Magnenat-Thalmann, "Made-tomeasure Technologies for Online Clothing Store", IEEE Computer Graphics and Applications, 2003,pp. 38-48.

[14] Baraff, A. Witkin, and M. Kass, "Untangling Cloth", Proceedings of ACM SIGGRAPH 2003, 2003, pp.862-870.

[15] A. Witkin, D. Baraff, "Differential Equation Basics", Proceedings of ACM SIGGRAPH 2001, 2001,pp.1-7.

[16] B. Witkin, "Particle System Dynamics", Proceedings of ACM SIGGRAPH 2001,2001,pp.8-19.

[17] P. Volino, N. Magnenat-Thalmann, "Accurate Collision response on polygonal Meshes", Computer Animation Conference, IEEE Publisher, 2000, pp. 154-163.

[18] Lv Qinan. On the materiality of teaching medium. Zhejiang Educational Technology.2011(05):10-14

[19] Ji Chengzhang. Design and Implementation of 3D Virtual Campus System of Weifang University of Science and Technology. Ocean University of China; Academic Dissertation. 2010:35-37.

(C) Jiang et al.; Licensee Bentham Open.

This is an open access article licensed under the terms of the Creative Commons Attribution Non-Commercial License (http://creativecommons.org/licenses/by-nc/3.0/) which permits unrestricted, non-commercial use, distribution and reproduction in any medium, provided the work is properly cited. 